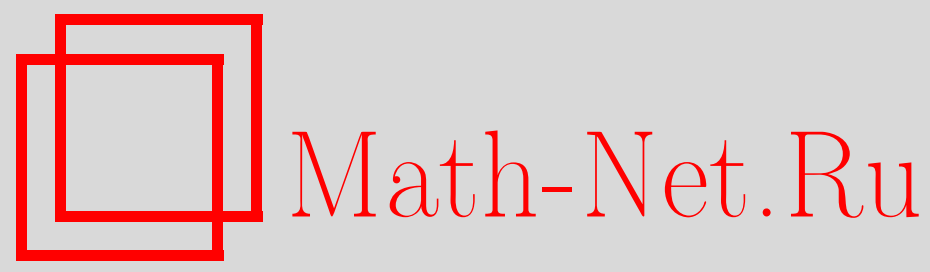

Э. Ю. Шамарова, Построение броуновского листа со значениями в компактном римановом многообразии, Матем. заметки, 2004, том 76, выпуск 4, 635-640

DOI: https://doi.org/10.4213/mzm578

Использование Общероссийского математического портала Math-Net.Ru подразумевает, что вы прочитали и согласны с пользовательским соглашением http://www.mathnet.ru/rus/agreement

Параметры загрузки:

IP : 3.85 .183 .62

26 апреля 2023 г., 13:10:29

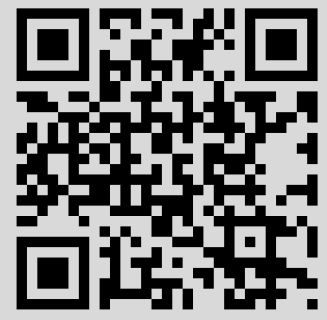




\section{ПОСТРОЕНИЕ БРОУНОВСКОГО ЛИСТА СО ЗНАЧЕНИЯМИ В КОМПАКТНОМ РИМАНОВОМ МНОГООБРАЗИИ}

\section{Э. Ю. Шамарова}

В настоящей работе предложен новый метод построения двупараметрического случайного поля $\mathbf{W}_{M}^{x}(s, t), x \in M$, со значениями в компактном римановом многообразии $M$, обладающего тем свойством, что случайные процессы $\mathbf{W}_{M}^{x}(\cdot, t)$ и $\mathbf{W}_{M}^{x}(s, \cdot)$ представляют собой броуновские движения на многообразии $M$ с параметрами $t$ и $s$ соответственно, начинающиеся в точке $x$ (под $6 p o-$ уновским движсением на многообразии $M$ с параметром $t$ понимается диффузионный процесс, порождаемый оператором $-(t / 2) \Delta_{M}$, где $\Delta_{M}$ - оператор Лапласа на многообразии $\left.M\right)$. В том случае, когда многообразие представляет собой компактную группу Ли, построенное двупараметрическое случайное поле совпадает с броуновским листом, определенным Малливеном в 1991 г. в работе [1] (сам Малливен называл это случайное поле броуновским движением со значениями в $C([0,1], M)$ - множестве непрерьвных функций, определенных на отрезке $[0,1]$ и принимающих значения в $M)$. Тем не менее, и в случае, когда многообразие является компактной группой Ли, метод, предложенный в настоящей работе, принципиально отличается от метода работы Малливена.

1. Первый шаг построения случайного поля $\mathbf{W}_{M}^{x}$. Пусть $M-d$-мерное компактное риманово многообразие без границы, изометрически вложенное в $\mathbb{R}^{m}$. Под броуновским листом со значениями в $\mathbb{R}^{m}$ мы понимаем семейство $m$ независимых стандартных броуновских листов. Пусть $\mathbf{W}_{t, s}-n$-мерный броуновский лист. Р ассмотрим $\mathbf{W}_{t, s}$ как процесс, принимающий значения в пространстве $C\left([0,1], \mathbb{R}^{m}\right)$. Обозначим этот процесс символом $\mathbf{W}_{t}$. Введем следующие обозначения: если $E$ - локально вьпуклое пространство (ЛВП), то $E^{t}$ означает $C([0, t], E)$; если $y \in C\left([0,1], \mathbb{R}^{m}\right)$ - некоторая непрерывная функция, то $\mathbb{W}^{y}$ означает распределение процесса $\mathbf{W}_{t}^{y}=y+\mathbf{W}_{t}$. Если $\psi \in C\left([0,1], \mathbb{R}^{m}\right)$, то определим процесс $\left(\mathbf{W}_{\psi}^{y}\right)_{t}=\psi(t)+\mathbf{W}_{t}^{y}$. Пусть $\widetilde{\mathbb{W}}_{\psi}^{y}-$ распределение этого процесса, $\mathrm{E}_{y, \psi}$ - математическое ожидание относительно меры $\widetilde{\mathbb{W}}_{\psi}^{y}$. Далее, $U_{\varepsilon}(M)$ означает $\varepsilon$-окрестность многообразия $M$. Мы будем рассматривать $\mathbf{W}_{\psi}^{y}$ для функций $y$ и $\psi$, удовлетворяющих условиям: $y(0) \in M, \psi(0)=0$. Цель этого пункта - доказать существование следующего ниже предела относительно семейства ограниченных непрерывных цилиндрических функций, где под цилиндрической функцией $C\left([0,1] \times[0,1], \mathbb{R}^{m}\right) \rightarrow \mathbb{R}$ мы всюду будем понимать такую функцию $f$, для которой найдется конечный набор точек $\tau_{1}, \ldots, \tau_{n}, \xi_{1}, \ldots, \xi_{k}$ и функция $\widetilde{f}: \mathbb{R}^{n k} \rightarrow \mathbb{R}$ такие, что

$$
f(\omega)=\widetilde{f}\left(\omega\left(\tau_{1}, \xi_{1}\right), \omega\left(\tau_{1}, \xi_{2}\right), \ldots, \omega\left(\tau_{n}, \xi_{k}\right)\right) .
$$

Этот предел определяет меру $\widetilde{\mathbb{W}}_{M, \psi, s, t}^{y}$ :

$$
\int_{C\left([0, s], \mathbb{R}^{m}\right)^{t}} f(\omega) \widetilde{\mathbb{W}}_{M, \psi, s, t}^{y}(d \omega)=\lim _{\varepsilon \rightarrow 0} \frac{\mathrm{E}_{y, \psi}\left\{f(\omega) \mathbb{I}_{\left\{\left(\mathbf{W}_{\psi}^{y}\right)_{t}(s) \in U_{\varepsilon}(M)\right\}}\right\}}{\widetilde{\mathbb{W}}_{\psi}^{y}\left\{\left(\mathbf{W}_{\psi}^{y}\right)_{t}(s) \in U_{\varepsilon}(M)\right\}} .
$$

Перед тем, как мы докажем существование этого предела, рассмотрим процесс

$$
\left(\mathbf{W}_{\psi, s}^{z}\right)_{t}=\psi(t)+B_{t}^{s}
$$

где $\psi:[0,1] \rightarrow \mathbb{R}^{m}$ - непрерьвная функция, удовлетворяющая условию $\psi(0)=0$, и $B_{t}^{s}-$ броуновское движение с параметром $s$, начинающееся в точке $z$. Результаты, полученные для этого процесса, будут применены для последующего построения.

Некоторые результаты для процесса $\left(\mathbf{W}_{\psi, s}^{z}\right)_{t}$. Пусть $\mathbb{W}_{\psi, s}^{z}$ означает распределение процесca $\left(\mathbf{W}_{\psi, s}^{z}\right)_{t}, \mathrm{E}_{z, \psi, s}$ - математическое ожидание относительно этого распределения. 
ЛЕмма 1. Предел

$$
\int_{C\left([0, t], \mathbb{R}^{m}\right)} f(\omega) \mathbb{W}_{M, \psi, s, t}^{z}(d \omega)=\lim _{\varepsilon \rightarrow 0} \frac{\mathrm{E}_{z, \psi, s}\left\{f(\omega) \mathbb{I}_{\left\{\left(\mathbf{W}_{\psi, s}^{z}\right)_{t} \in U_{\varepsilon}(M)\right\}}\right\}}{\mathbb{W}_{\psi, s}^{z}\left\{\left(\mathbf{W}_{\psi, s}^{z}\right)_{t} \in U_{\varepsilon}(M)\right\}}
$$

рассматриваемый относительно семейства непрерывных ограниченных чилиндрических функций, существует и определяет меру $\mathbb{W}_{M, \psi, s, t}^{z}$, стоящ,ую в интеграле слева.

ИДЕЯ ДоКАЗАТЕЛЬСТВА. Найдем функцию $\widetilde{f}: \mathbb{R}^{k+1} \rightarrow \mathbb{R}$ и конечное множество точек $\tau_{1}, \ldots$, $\tau_{k}$ такие, что

$$
f(\omega)=\widetilde{f}\left(\omega\left(\tau_{1}\right), \ldots, \omega\left(\tau_{k}\right), \omega(t)\right)
$$

Имеем

$$
\begin{gathered}
\int_{C\left([0, t], \mathbb{R}^{m}\right)} f(\omega) \mathbb{W}_{M, \psi, s, t}^{z}(d \omega)=\lim _{\varepsilon \rightarrow 0} \frac{\int_{C\left([0, t], \mathbb{R}^{m}\right)} f(\omega) \mathbb{I}_{\left\{\omega: \omega(t) \in U_{\varepsilon}(M)\right\}} \mathbb{W}_{\psi, s}^{z}(d \omega)}{\mathbb{W}_{\psi, s}^{z}\left\{\omega: \omega(t) \in U_{\varepsilon}(M)\right\}} \\
=\lim _{\varepsilon \rightarrow 0} \frac{1}{\mathrm{P}^{\mathbb{W}}\left(t, 0, U_{\varepsilon}(M-z-\psi(t))\right)} \int_{\mathbb{R}^{m}} \mathrm{P}^{\mathbb{W}}\left(\tau_{1}, 0, d x_{1}\right) \int_{\mathbb{R}^{m}} \mathrm{P}^{\mathbb{W}}\left(\tau_{2}-\tau_{1}, x_{1}, d x_{2}\right) \cdots \\
\quad \times \int_{U_{\varepsilon}(M-\psi(t)-z)} \mathrm{P}^{\mathbb{W}}\left(t-\tau_{k}, x_{k}, d x_{k+1}\right) \\
\times \widetilde{f}\left(x_{1}+z+\psi\left(\tau_{1}\right), \ldots, x_{k}+z+\psi\left(\tau_{k}\right), x_{k+1}+z+\psi(t)\right),
\end{gathered}
$$

где

$$
\mathrm{P}^{\mathbb{W}}(\tau, x, d z)=\frac{1}{(2 \pi s \tau)^{m / 2}} \exp \left\{-\frac{|z-x|^{2}}{2 s \tau}\right\} d z .
$$

Так как функция под знаком интеграла ограничена, то по теореме Лебега достаточно доказать существование предела

$$
\begin{aligned}
& \lim _{\varepsilon \rightarrow 0} \frac{\int_{\left(U_{\varepsilon}(M-\psi(t)-z)\right)} \tilde{f}\left(x_{1}+z+\psi\left(\tau_{1}\right), \ldots, x_{k+1}+z+\psi(t)\right) \mathrm{P}^{\mathbb{W}}\left(t-\tau_{k}, x_{k}, d x_{k+1}\right)}{\mathrm{P}^{\mathbb{W}}\left(t, 0, U_{\varepsilon}(M-z-\psi(t))\right)} \\
& =\lim _{\varepsilon \rightarrow 0} \frac{\int_{U_{\varepsilon}\left(M-\psi(t)-z-x_{k}\right)} \tilde{f}\left(x_{1}+z+\psi\left(\tau_{1}\right), \ldots, x_{k+1}+x_{k}+z+\psi(t)\right) \mathrm{P}^{\mathbb{W}}\left(t-\tau_{k}, 0, d x_{k+1}\right)}{\mathrm{P}^{\mathbb{W}}\left(t, 0, U_{\varepsilon}(M-z-\psi(t))\right.} .
\end{aligned}
$$

Обозначим через $M_{1}$ многообразие $M-\psi(t)-z-x_{k}$, через $M_{2}$ многообразие $M-\psi(t)-z$. Пусть далее

$$
\lambda_{\varepsilon}=\left.\frac{1}{\operatorname{vol}_{m-d}(\varepsilon)} l\right|_{U_{\varepsilon}\left(M_{1}\right)}, \quad \mu_{\varepsilon}=\left.\frac{1}{\operatorname{vol}_{m-d}(\varepsilon)} l\right|_{U_{\varepsilon}\left(M_{2}\right)}
$$

где $l$ - мера Лебега на $\mathbb{R}^{m}$. Легко видеть, что доказательство существования этого предела сводится к доказательству существования предела вида

$$
\lim _{\varepsilon \rightarrow 0} \frac{\int_{\mathbb{R}^{m}} g\left(x_{k+1}\right) \exp \left\{-\frac{\left|x_{k+1}-x_{k}\right|^{2}}{2 s\left(t-\tau_{k}\right)}\right\} \lambda_{\varepsilon}\left(d x_{k+1}\right)}{\int_{\mathbb{R}^{m}} \exp \left\{-\frac{\left|x_{k+1}\right|^{2}}{2 s t}\right\} \mu_{\varepsilon}\left(d x_{k+1}\right)}
$$

где $g: \mathbb{R} \rightarrow \mathbb{R}$ - другое обозначение для функции $\widetilde{f}$ в котором выражена зависимость только от последней переменной. Легко показать, что при $\varepsilon \rightarrow 0$ меры $\lambda_{\varepsilon}$ и $\mu_{\varepsilon}$ сходятся слабо к поверхностным мерам на $M_{1}$ и $M_{2}$ соответственно.

ЛЕмма 2. Предел (1) существует относительно семейства непрерывных ограниченньх иилиндрических функиий. 
ИДЕЯ ДОКАЗАТЕЛЬСТВА. Пусть $\mathrm{P}^{\widetilde{\mathbb{W}}}(t, y, \Gamma)=\widetilde{\mathbb{W}}^{y}(\omega: \omega(t) \in \Gamma)$ - переходная вероятность для меры $\widetilde{\mathbb{W}}^{y}$, где $y \in C\left([0,1], \mathbb{R}^{m}\right)$. Пусть далее функция $\widetilde{f}: C\left([0, s], \mathbb{R}^{m}\right)^{k+1} \rightarrow \mathbb{R}$ и конечное множество точек $\tau_{1}, \tau_{2}, \ldots, \tau_{k}$ таковы, что

$$
f(\omega)=\widetilde{f}\left(\omega\left(\tau_{1}\right), \omega\left(\tau_{2}\right), \ldots, \omega\left(\tau_{k}\right), \omega(t)\right) .
$$

Символ $\pi_{s}$ означает координатное отображение. Доказательство проводится с использованием формулы из [2, с. 204]

$$
\begin{aligned}
& \int_{C\left([0, s], \mathbb{R}^{m}\right)^{t}} f(\omega) \widetilde{\mathbb{W}}^{0}(d \omega)=\int_{C\left([0, s], \mathbb{R}^{m}\right)} \mathrm{P}^{\widetilde{\mathbb{W}}}\left(\tau_{1}, 0, d w_{1}\right) \int_{C\left([0, s], \mathbb{R}^{m}\right)} \mathrm{P}^{\widetilde{\mathbb{W}}}\left(\tau_{2}-\tau_{1}, w_{1}, d w_{2}\right) \cdots \\
& \quad \times \int_{\pi_{s}^{-1}\left(U_{\varepsilon}(M-\psi(t)-y(s))\right)} \widetilde{f}\left(w_{1}, \ldots, w_{k+1}\right) \mathrm{P}^{\widetilde{\mathbb{W}}}\left(t-\tau_{k}, w_{k}, d w_{k+1}\right)
\end{aligned}
$$

и применением леммы 1 к мере в последнем интеграле.

\section{2. Асимптотика по $t$ для интеграла одного вида.}

ПРЕДЛОЖЕнИЕ 1. Пусть $i$ - изометрическое вложение многообразия $M$ в $\mathbb{R}^{m}, g \in$ $C^{2}(M)$. Тогда

$\frac{1}{(2 \pi t)^{d / 2}} \int_{M} g(z) \exp \left\{-\frac{|z-y|^{2}}{2 t}\right\} \lambda_{M}(d z)=g(y)+\frac{t}{8} g(y)(c(y)-\operatorname{scal}(y))-\frac{t}{2} \Delta_{M} g(y)+t R(t, y)$, где $|R(t, y)|<K t^{1 / 2}, K-$ константа, которая не зависит от $y, \operatorname{scal}(y)-$ скалярная кривизна в точке $y$; функиия $c(y)$ имеет вид

$$
c(y)=\sum_{k, l} \sum_{\alpha}\left(\frac{\partial^{2} i^{\alpha}}{\partial x^{k} \partial x^{l}}\right)^{2}(0),
$$

где $x^{k}$ - нормальные координаты в некоторой окрестности $U_{y}$ точки $y$, обеспечиваемые гомеоморфизмом окрестности $U_{y}$ на некоторую окрестность нуля $U$ в $\mathbb{R}^{d}$. Независимо от локальных координат $c(y)$ может быть записана в виде

$$
c(y)=-\left.\frac{1}{2} \Delta_{M} \Delta_{M}|y-\cdot|^{2}\right|_{y}-\frac{1}{3} \operatorname{scal}(y)
$$

и, следовательно, $с(y)$ зависит только от вложения $i$.

ИДЕЯ ДОКАЗАТЕЛЬСТВА. Получено более точное асимптотическое выражение по сравнению с тем, которое было получено для интеграла такого же вида в [3]. Идея доказательства та же самая.

Следствие 1. Пусть $g \in C^{2}(M)$. Тогда мы имеем следующую асимптотику:

$$
\frac{\int_{M} g(z) \exp \left\{-\frac{|z-y|^{2}}{2 t}\right\} \lambda_{M}(d z)}{\int_{M} \exp \left\{-\frac{|z-y|^{2}}{2 t}\right\} \lambda_{M}(d z)}=g(y)-\frac{t}{2} \Delta_{M} g(y)+t R_{1}(t, y),
$$

əде $\left|R_{1}(t, y)\right|<K_{1} t^{1 / 2}$, а $K_{1}$ - константа, не зависящая от $y$.

СледСтвиЕ 2. Пусть $g \in C^{2}(M), y \in M, \psi-$ гёльдерова функция порядка гёльдеровости $1 / 3<\alpha<1 / 2$ такая, что $\psi(0)=0$. Пусть $\operatorname{Pr}_{M}$ - отображение проектирования на многообразие $M$ вдоль нормальных $к$ многообразию подпространств, определенное в подходящей окрестности многообразия, $\psi_{M}(t, y)=\operatorname{Pr}_{M}(y+\psi(t))$. Имеет место следующая асимптотика:

$$
\frac{\int_{M} g(z) \exp \left\{-\frac{|z-y-\psi(t)|^{2}}{2 t}\right\} \lambda_{M}(d z)}{\int_{M} \exp \left\{-\frac{|z-y-\psi(t)|^{2}}{2 t}\right\} \lambda_{M}(d z)}=g\left(y+\psi_{M}(t)\right)-\frac{t}{2} \Delta_{M} g(y)+t R_{2}(t, y),
$$

əде $\left|R_{2}(t, y)\right|<K_{2} t^{3 \alpha-1}, K_{2}-$ константа. 
3. Второй шаг построения случайного поля $\mathbf{W}_{M}^{x}$. Пусть $f$-непрерывная ограниченная цилиндрическая функция на $C\left([0, s], \mathbb{R}^{m}\right)^{1}, \varphi: \mathbb{R} \rightarrow M-$ функция, являющаяся траекторией броуновского движения на $M$, такая, что $\varphi(0)=x$. Пусть $\mathscr{P}_{1}=\left\{0=t_{0} \leqslant t_{1} \leqslant \cdots \leqslant t_{n}=1\right\}-$ разбиение интервала $[0,1]$. Если $E$ - это ЛВП, то каждое $\omega \in E^{1}$ может быть отождествлено с конечной последовательностью из $n$ элементов

$$
\left(\omega_{1}, \omega_{2}, \ldots, \omega_{n}\right) \in E^{t_{1}} \times E^{t_{2}-t_{1}} \times \cdots \times E^{t_{n}-t_{n-1}},
$$

где $\omega_{j}$ определено на интервале $\left[0, t_{j}-t_{j-1}\right]$ по формуле $\omega_{j}(t)=\omega\left(t_{j-1}+t\right)$. Определим функцию $\varphi_{t_{i-1}} t_{i}$ на интервале $\left[0, t_{i}-t_{i-1}\right]$ следующим образом:

$$
\varphi_{t_{i-1} t_{i}}(t)=\varphi\left(t_{i-1}+t\right)-\varphi\left(t_{i-1}\right) .
$$

Определим меру $\widetilde{\mathbb{W}}_{M, \varphi, s, \mathscr{P}_{1}}^{x}$ формулой

$$
\begin{aligned}
& \int_{C\left([0, s], \mathbb{R}^{m}\right)^{1}} f(\omega) \widetilde{\mathbb{W}}_{M, \varphi, s, \mathscr{P}_{1}}^{x}(d \omega)=\int_{C\left([0, s], \mathbb{R}^{m}\right)^{t_{1}}} \widetilde{\mathbb{W}}_{M, \varphi_{0 t_{1}}, s, t_{1}}^{x}\left(d \omega_{1}\right) \\
& \quad \times \int_{C\left([0, s], \mathbb{R}^{m}\right)^{t_{2}-t_{1}}} \widetilde{\mathbb{W}}_{M, \varphi_{t_{1} t_{2}}, s, t_{2}-t_{1}}^{\omega_{1}\left(t_{1}\right)}\left(d \omega_{2}\right) \cdots \\
& \quad \times \int_{C\left([0, s], \mathbb{R}^{m}\right)^{t_{n}-t_{n-1}}} \widetilde{\mathbb{W}}_{M, \varphi_{t_{n-1} t_{n}, s, t_{n}-t_{n-1}}^{\omega_{n-1}\left(t_{n-1}-t_{n-2}\right)}\left(d \omega_{n}\right) f\left(\omega_{1}, \omega_{2}, \ldots, \omega_{n}\right) .}
\end{aligned}
$$

Непосредственно проверяется, что $\omega_{i}\left(t_{i}-t_{i-1}\right)(0) \in M$, так что мера $\widetilde{\mathbb{W}}_{M, \varphi, s, \mathscr{P}_{1}}^{x}$ определена корректно. Далее, пусть $\mathscr{P}_{2}=\left\{0=s_{0} \leqslant s_{1} \leqslant \cdots \leqslant s_{k}=1\right\}$ - разбиение интервала [0,1]. Пусть теперь $s$ - параметр времени. Вместо символа $\widetilde{\mathbb{W}}_{M, \varphi, s, \mathscr{P}_{1}}^{x}$ будем пользоваться символом $\widetilde{\mathbb{W}}_{M, s, \mathscr{P}_{1}}^{\varphi} \cdot$ Определим меру $\mathbb{W}_{M, \mathscr{P}_{1}, \mathscr{P}_{2}}^{x}$ формулой

$$
\begin{aligned}
& \int_{C\left([0,1], \mathbb{R}^{m}\right)^{1}} f(\omega) \mathbb{W}_{M, \mathscr{P}_{1}, \mathscr{P}_{2}}^{x}(d \omega)=\int_{C\left([0,1], \mathbb{R}^{m}\right)^{s_{1}}} \widetilde{\mathbb{W}}_{M, s_{1}, \mathscr{P}_{1}}^{x}\left(d \omega_{1}\right) \\
& \times \int_{C\left([0,1], \mathbb{R}^{m}\right)^{s_{2}-s_{1}}} \widetilde{\mathbb{W}}_{M, s_{2}-s_{1}, \mathscr{P}_{1}}^{\omega_{1}\left(s_{1}\right)}\left(d \omega_{2}\right) \cdots \\
& \times \int_{C\left([0,1], \mathbb{R}^{m}\right)^{s_{n}-s_{n-1}}} \widetilde{\mathbb{W}}_{M, s_{n}-s_{n-1}, \mathscr{P}_{1}}^{\omega_{n-1}\left(s_{n-1}-s_{n-2}\right)}\left(d \omega_{n}\right) f\left(\omega_{1}, \ldots, \omega_{n}\right) .
\end{aligned}
$$

Теорема 1. Для каждого $x \in M$, если мелкости разбиений $\mathscr{P}_{1}$ и $\mathscr{P}_{2}$ стремятся кнулю, то последовательность мер $\mathbb{W}_{M, \mathscr{P}_{1}, \mathscr{P}_{2}}^{x}$ сходится слабо относительно семейства непрерывных ограниченных чилиндрических функиий кмере $\mathbb{W}_{M}^{x}$. Мера $\mathbb{W}_{M}^{x}$, рассматриваемая как распределение прочесса со значениями в $C([0,1], M)$, обладает переходной вероятностью в момент времени $t$, совпадающей с распределением броуновского движения на многообразии с параметром $t$, начинающегося в точке $x$.

ИДЕЯ ДОКАЗАТЕЛЬСТВА. Пусть

$$
\begin{aligned}
h \cdot \mathbb{W}_{M, \varphi, s, \mathscr{P}_{1}}^{x}\left(=h \cdot \mathbb{W}_{M, s, \mathscr{P}_{1}}^{\varphi}\right)=\int_{C\left([0,1], \mathbb{R}^{m}\right)} h(\omega) \mathbb{W}_{M, \varphi, s, \mathscr{P}_{1}}^{x}(d \omega) \\
=\int_{C\left(\left[0, t_{1}\right], \mathbb{R}^{m}\right)} \mathbb{W}_{M, \varphi_{0 t_{1}}, s, t_{1}}^{x}\left(d \omega_{1}\right) \int_{C\left(\left[0, t_{2}-t_{1}\right], \mathbb{R}^{m}\right)} \mathbb{W}_{M, \varphi_{t_{1} t_{2}, s, t_{2}-t_{1}}\left(d \omega_{2}\right) \cdots}^{\omega_{1}\left(t_{1}\right)} \\
\quad \times \int_{C\left(\left[0, t_{n}-t_{n-1}\right], \mathbb{R}^{m}\right)} \mathbb{W}_{M, \varphi_{t_{n-1} t_{n}, s, t_{n}-t_{n-1}}^{\omega_{n-1}\left(t_{n-1}-t_{n-2}\right)}\left(d \omega_{n}\right) h\left(\omega_{1}, \omega_{2}, \ldots, \omega_{n}\right) .}
\end{aligned}
$$

Пусть существует $\tilde{f}: C\left([0,1], \mathbb{R}^{m}\right) \rightarrow \mathbb{R}$ такая, что $f(\omega)=\widetilde{f}(\omega(t))$. Тогда

$$
\begin{aligned}
\int_{C\left([0, s], \mathbb{R}^{m}\right)^{1}} f(\omega) \widetilde{\mathbb{W}}_{M, s, \mathscr{P}_{1}}^{\varphi}(d \omega) & =\int_{C\left([0,1], \mathbb{R}^{m}\right)} \tilde{f}(w) \widetilde{\mathbb{W}}_{M, s, \mathscr{P}_{1}}^{\varphi} \circ \pi_{s}^{-1}(d w) \\
& =\int_{C\left([0,1], \mathbb{R}^{m}\right)} \tilde{f}(w) \mathbb{W}_{M, s, \mathscr{P}_{1}}^{\varphi}(d w) .
\end{aligned}
$$


Отсюда следует

$$
\begin{aligned}
& \int_{C\left([0,1], \mathbb{R}^{m}\right)^{1}} f(\omega) \mathbb{W}_{M, \mathscr{P}_{1}, \mathscr{P}_{2}}^{x}(d \omega)=\int_{C\left([0,1], \mathbb{R}^{m}\right)} \mathbb{W}_{M, s_{1}, \mathscr{P}_{1}}^{x}\left(d w_{1}\right) \\
& \quad \times \int_{C\left([0,1], \mathbb{R}^{m}\right)} \mathbb{W}_{M, s_{2}-s_{1}, \mathscr{P}_{1}}^{w_{1}}\left(d w_{2}\right) \cdots \int_{C\left([0,1], \mathbb{R}^{m}\right)} \mathbb{W}_{M, s_{n-1}-s_{n-2}, \mathscr{P}_{1}}^{w_{n-2}}\left(d w_{n-1}\right) \\
& \quad \times \int_{C\left([0,1], \mathbb{R}^{m}\right)} \mathbb{W}_{M, s_{n}-s_{n-1}, \mathscr{P}_{1}}^{w_{n-1}}\left(d w_{n}\right) \widetilde{f}\left(w_{n}\right) .
\end{aligned}
$$

Рассмотрим интеграл вида

$$
\int_{C\left([0, t], \mathbb{R}^{m}\right)} g(\omega) \mathbb{W}_{M, \psi, s, t}^{z}(d \omega)
$$

где функция $g \in C\left([0, t], \mathbb{R}^{m}\right)$ такова, что существует функция $\widetilde{g} \in C(\mathbb{R})$ такая, что $g(\omega)=\widetilde{g}(\omega(t))$. В результате несложных вычислений получим

$$
\begin{aligned}
\int_{C\left([0, t], \mathbb{R}^{m}\right)} g(\omega) \mathbb{W}_{M, \psi, s, t}^{z}(d \omega) & =\lim _{\varepsilon \rightarrow 0} \frac{\int_{C\left([0, t], \mathbb{R}^{m}\right)} g(\omega) \mathbb{I}_{\left\{\omega: \omega(t) \in U_{\varepsilon}(M)\right\}}(\omega) \mathbb{W}_{\psi, s}^{z}(d \omega)}{\mathbb{W}_{\psi, s}^{z}\left\{\omega: \omega(t) \in U_{\varepsilon}(M)\right\}} \\
& =\frac{\int_{M} \exp \left\{-\frac{\left|x_{1}-z-\psi(t)\right|^{2}}{2 t s}\right\} \widetilde{g}\left(x_{1}\right) \lambda_{M}\left(d x_{1}\right)}{\int_{M} \exp \left\{-\frac{\left|x_{1}-z-\psi(t)\right|^{2}}{2 t s}\right\} \lambda_{M}\left(d x_{1}\right)} .
\end{aligned}
$$

Предположим сначала, что функция $f$ такова, что найдутся функция $p: \mathbb{R}^{m} \rightarrow \mathbb{R}$ и числа $t, s \in$ $[0,1]$, такие, что $f(\omega)=p(\omega(t, s))$. Интеграл

имеет вид

$$
\int_{C\left([0,1], \mathbb{R}^{m}\right)^{1}} f(\omega) \mathbb{W}_{M, \mathscr{P}_{1}, \mathscr{P}_{2}}^{x}(d \omega)
$$

$$
\begin{aligned}
& \frac{\int_{M} \exp \left\{-\frac{\left|x_{1}-x\right|^{2}}{2 \Delta s_{1} \Delta t_{1}}\right\} d x_{1}}{\int_{M} \exp \left\{-\frac{\left|\bar{x}_{1}-x\right|^{2}}{2 \Delta s_{1} \Delta t_{1}}\right\} d \bar{x}_{1}} \cdots \frac{\int_{M} \exp \left\{-\frac{\left|x_{n-1}-x_{n-2}\right|^{2}}{2 \Delta s_{1} \Delta t_{n-1}}\right\} d x_{n-1}}{\int_{M} \exp \left\{-\frac{\left|\bar{x}_{n-1}-x_{n-2}\right|^{2}}{2 \Delta s_{1} \Delta t_{n-1}}\right\} d \bar{x}_{n-1}} \frac{\int_{M} \exp \left\{-\frac{\left|x_{n}-x_{n-1}\right|^{2}}{2 \Delta s_{1} \Delta t_{n}}\right\} d x_{n}}{\int_{M} \exp \left\{-\frac{\left|\bar{x}_{n}-x_{n-1}\right|^{2}}{2 \Delta s_{1} \Delta t_{n}}\right\} d \bar{x}_{n}} \\
& \times \frac{\int_{M} \exp \left\{-\frac{\left|y_{1}-x_{1}\right|^{2}}{2 \Delta s_{2} \Delta t_{1}}\right\} d y_{1}}{\int_{M} \exp \left\{-\frac{\left|\bar{y}_{1}-x_{1}\right|^{2}}{2 \Delta s_{2} \Delta t_{1}}\right\} d \bar{y}_{1}} \cdots \frac{\int_{M} \exp \left\{-\frac{\left|y_{n-1}-y_{n-2}-x_{n-1}+x_{n-2}\right|^{2}}{2 \Delta s_{2} \Delta t_{n-1}}\right\} d y_{n-1}}{\int_{M} \exp \left\{-\frac{\left|\bar{y}_{n-1}-y_{n-2}-x_{n-1}+x_{n-2}\right|^{2}}{2 \Delta s_{2} \Delta t_{n-1}}\right\} d \bar{y}_{n-1}} \\
& \times \frac{\int_{M} \exp \left\{-\frac{\left|y_{n}-y_{n-1}-x_{n}+x_{n-1}\right|^{2}}{2 \Delta s_{2} \Delta t_{n}}\right\} d y_{n}}{\int_{M} \exp \left\{-\frac{\left|\bar{y}_{n}-y_{n-1}-x_{n}+x_{n-1}\right|^{2}}{2 \Delta s_{2} \Delta t_{n}}\right\} d \bar{y}_{n}} \cdots \\
& \times \frac{\int_{M} \exp \left\{-\frac{\left|u_{1}-z_{1}\right|^{2}}{2 \Delta s_{k-1} \Delta t_{1}}\right\} d u_{1}}{\int_{M} \exp \left\{-\frac{\left|\bar{u}_{1}-z_{1}\right|^{2}}{2 \Delta s_{k-1} \Delta t_{1}}\right\} d \bar{u}_{1}} \cdots \frac{\int_{M} \exp \left\{-\frac{\left|u_{n-1}-u_{n-2}-z_{n-1}+z_{n-2}\right|^{2}}{2 \Delta s_{k-1} \Delta t_{n-1}}\right\} d u_{n-1}}{\int_{M} \exp \left\{-\frac{\left|\bar{u}_{n-1}-u_{n-2}-z_{n-1}+z_{n-2}\right|^{2}}{2 \Delta s_{k-1} \Delta t_{n-1}}\right\} d \bar{u}_{n-1}} \\
& \times \frac{\int_{M} \exp \left\{-\frac{\left|u_{n}-u_{n-1}-z_{n}+z_{n-1}\right|^{2}}{2 \Delta s_{k-1} \Delta t_{n}}\right\} d u_{n}}{\int_{M} \exp \left\{-\frac{\left|\bar{u}_{n}-u_{n-1}-z_{n}+z_{n-1}\right|^{2}}{2 \Delta s_{k-1} \Delta t_{n}}\right\} d \bar{u}_{n}} \\
& \times \frac{\int_{M} \exp \left\{-\frac{\left|v_{1}-u_{1}\right|^{2}}{2 \Delta s_{k} \Delta t_{1}}\right\} d v_{1}}{\int_{M} \exp \left\{-\frac{\left|\bar{v}_{1}-u_{1}\right|^{2}}{2 \Delta s_{k} \Delta t_{1}}\right\} d \bar{v}_{1}} \cdots \frac{\int_{M} \exp \left\{-\frac{\left|v_{n-1}-v_{n-2}-u_{n-1}+u_{n-2}\right|^{2}}{2 \Delta s_{k} \Delta t_{n-1}}\right\} d v_{n-1}}{\int_{M} \exp \left\{-\frac{\left|\bar{v}_{n-1}-v_{n-2}-u_{n-1}+u_{n-2}\right|^{2}}{2 \Delta s_{k} \Delta t_{n-1}}\right\} d \bar{v}_{n-1}} \\
& \times \frac{\int_{M} \exp \left\{-\frac{\left|v_{n}-v_{n-1}-u_{n}+u_{n-1}\right|^{2}}{2 \Delta s_{k} \Delta t_{n}}\right\} p\left(v_{n}\right) d v_{n}}{\int_{M} \exp \left\{-\frac{\left|\bar{v}_{n}-v_{n-1}-u_{n}+u_{n-1}\right|^{2}}{2 \Delta s_{k} \Delta t_{n}}\right\} d \bar{v}_{n}},
\end{aligned}
$$


где $\Delta t_{i}=t_{i}-t_{i-1}, \Delta s_{j}=s_{j}-s_{j-1}$, для упрощения обозначений вместо $\lambda_{M}(d z)$ использовано обозначение $d z$. Мы также считали, что $t_{n}=t, s_{k}=s$. Обозначим этот интеграл через $I\left(\mathscr{P}_{1}, \mathscr{P}_{2}, p\right)$.

Лемма 3. Интеграл $I\left(\mathscr{P}_{1}, \mathscr{P}_{2}, p\right)$ сходится $\kappa \exp \left\{-\frac{s t}{2} \Delta_{M}\right\} p$, если мелкости $\left|\mathscr{P}_{1}\right|$ u $\left|\mathscr{P}_{2}\right|$ стремятся $\propto$ нулю.

ИДЕЯ ДОКАЗАТЕЛЬСТВА. С использованием следствий 1 и 2 предложения 1 получаем следующую асимптотику для интеграла $I\left(\mathscr{P}_{1}, \mathscr{P}_{2}, p\right)$ :

$$
I\left(\mathscr{P}_{1}, \mathscr{P}_{2}, p\right)(x)=p(x)-\frac{s t}{2} \Delta_{M} p(x)+O\left(s^{2} t^{2}\right)+O\left(\left|\mathscr{P}_{2}\right|,\left|\mathscr{P}_{1}\right|\right) .
$$

Откуда следует, что существует предел

$$
\lim _{\left|\mathscr{P}_{1}\right| \rightarrow 0,\left|\mathscr{P}_{2}\right| \rightarrow 0} I\left(\mathscr{P}_{1}, \mathscr{P}_{2}, p\right)(x)=p(x)-\frac{s t}{2} \Delta_{M} p(x)+O\left(s^{2} t^{2}\right)\left(=\left(Q_{s t} p\right)(x)\right) .
$$

Проверим далее, что $Q_{\tau+\Delta \tau}=Q_{\tau} Q_{\Delta \tau}$. Пусть $s$ и $t$ таковы, что $\tau=s t$. Найдем $\Delta s$ и $\Delta t$ такие, что $\tau+\Delta \tau=(s+\Delta s)(t+\Delta t)$, и рассмотрим интеграл $I\left(\mathscr{P}_{1}^{[0, t+\Delta t]}, \mathscr{P}_{2}^{[0, s+\Delta s]}, p\right)$ для разбиений отрезков $[0, s+\Delta s]$ и $[0, t+\Delta t]$, дополнив соответствующие разбиения отрезков $[0, s]$ и $[0, t]$ точками разбиений отрезков $[t, t+\Delta t],[s, s+\Delta s]$. Из структуры интеграла $I\left(\mathscr{P}_{1}, \mathscr{P}_{2}, p\right)$ легко видеть, что

$$
I\left(\mathscr{P}_{1}^{[0, t+\Delta t]}, \mathscr{P}_{2}^{[0, s+\Delta s]}, p\right)=I\left(\mathscr{P}_{1}^{[0, t]}, \mathscr{P}_{2}^{[0, s]}, I\left(\mathscr{P}_{1}^{[t, t+\Delta t]}, \mathscr{P}_{2}^{[s, s+\Delta s]}, p\right)\right) .
$$

Переходя к пределу в этом выражении и учитьвая, что $\Delta \tau=s \Delta t+t \Delta s+\Delta s \Delta t$, мы получим, что $Q_{\tau}$ является полугруппой, для которой в силу доказанного выше справедливо

$$
\left(Q_{\tau} p\right)(x)=p(x)-\frac{\tau}{2} \Delta_{M} p(x)+O\left(\tau^{2}\right) .
$$

Таким образом, мы заключаем, что

$$
Q_{\tau}=\exp \left\{-\frac{\tau}{2} \Delta_{M}\right\}
$$

Для функции $f$, зависящей от $\omega$ в нескольких точках, скажем, в точках $\xi_{i} \in[0, s]$ и $\tau_{j} \in[0, t]$, вид интеграла $I\left(\mathscr{P}_{1}, \mathscr{P}_{2}, p\right)$ будет таким же. Сходимость, рассмотренная выше, будет иметь место на каждом квадрате $\left[\xi_{i-1}, \xi_{i}\right] \times\left[\tau_{j-1}, \tau_{j}\right]$. Каждый из операторов вида

$$
\exp \left\{-\frac{\Delta \xi_{i} \Delta \tau_{j}}{2} \Delta_{M}\right\}
$$

действует на соответствующую переменную функции $p$, определенную как

$$
f(\omega)=p\left(\omega_{11}\left(\xi_{1}, \tau_{1}\right), \ldots, \omega_{k l}\left(\xi_{l}, \tau_{l}\right)\right)
$$

где $\omega_{i j}$ определена на $\left[0, \xi_{i}-\xi_{i-1}\right] \times\left[0, \tau_{j}-\tau_{j-1}\right]$ по формуле $\omega_{i j}(s, t)=\omega\left(\xi_{i-1}+s, \tau_{j-1}+t\right)$.

СледствиЕ 3. Пусть $M-$ компактная группа Ли. Тогда $\mathbf{W}_{M}^{x}$, рассматриваемъи й как прочесс со значениями в $C([0,1], M)$, совпадает с броуновским движением, построенным $\boldsymbol{\theta}[1]$.

ИДЕЯ ДОКАЗАТЕЛЬСТВА. Доказательство следует из теоремы 1 и теоремы 2.15 из статьи [4] (теорема 2.15 из [4] доказана также в лемме 3.3 в статье [5]).

\section{СПИСОК ЦИТИРОВАННОЙ ЛИТЕРАТУРЫ}

1. Malliavin P. // Diffusion Processes and Related Problems in Analysis (Evanston, IL, 1989). V.1. Boston, MA: Birkhäuser, 1990. P. 17-31. 2. Ikeda N., Watanabe Sh. Stochastic Differential Equations and Diffusion Processes. Amsterdam: North-Holland, 1981. 3. Smolyanov O. G., von Weizsäcker H., Wittich O. // Canad. Math. Soc. Conference Proc. 2000. V. 29. P. 589-602. 4. Driver B. K., Srimurthy V. K. // Ann. Probab. 2001. V. 29. № 2. P. 691-723. 5. Srimurthy V. K. // Probab. Theory Related Fields. 2000. V. 118. № 4. P. 522-546. 